\title{
Development of a Pellet Catalytic Combustor for a Small Scale Generation System Using Near Infrared Ray Irradiation*
}

\author{
Go OHMURA $^{* *}$, Tatsuya KEIDA ${ }^{* *}$, Takehiko SEO ${ }^{* * *}$, Chulju AHN ${ }^{* *}$, \\ Fumiteru AKAMATSU ${ }^{* *}$ and Masahiko SHIBAHARA ${ }^{* *}$ \\ **Department of Mechanical Engineering, Osaka University, \\ 2-1 Yamadaoka, Suita, Osaka 565-0871 \\ E-mail: siba@mech.eng.osaka-u.ac.jp \\ ***Department of Mechanical and Aerospace Engineering, Tokyo Institute of Technology, \\ 2-12-1 Ookayama, Meguro-ku, Tokyo 152-8550
}

\begin{abstract}
Several geometric types of the small scale catalytic combustors applying the pellet catalysts as both the heat source and the emitter for infrared ray generation were developed in order to investigate the catalytic combustion phenomenon and the effective operation conditions for the TPV generation system. The results of temperature measurements and exhaust gas analyses showed that there were the optimal width and length of the combustor geometry for the high combustion efficiency and the reduction of the residual $\mathrm{CO}$ concentration. The ultraviolet and visible spectral measurements showed that the surface chemical reaction was predominant in the catalytic combustion and the gas phase reaction which causes higher temperature and higher reaction rate than those of the surface reaction scarcely occurred in the developed combustor. The near infrared spectral measurements showed that the pellet catalysts in the combustor could play the role of the emitter for the TPV generation system.
\end{abstract}

Key words: Small Scale Combustor, Catalytic Combustion, TPV Generation, Near Infrared Ray, Spectral Measurement

\section{Introduction}

Due to the developments of portable electronic devices / appliances, a small electric generation system, driven by hydrocarbon fuels is expected as a promising high density generation system instead of conventional rechargeable batteries [1]. In particular, a thermophotovoltaic (TPV) generation system, which converts radiated infrared ray to electric power, is developed by many research groups, because of a lot of advantages such as high reliability as well as little demerits of the size reduction [2-5]. In the present research, to develop a small scale and high intensity heat source and to establish the method of generating near infrared ray (NIR) radiation for a TPV generation system, we proposed a new concept of the TPV generation system; the feature of the system is a small scale catalytic combustor applying the sphere-shaped pellet catalysts, which generate NIR as heat source / emitter and need lower activation energy for the combustion reaction. The discussions were conducted through the experimental results on what is the governing phenomenon on each component of the TPV generation system, mainly a catalytic combustor, and whether the proposed concept is useful for the TPV generating system or 
not.

In the present study, we conducted such experiments as direct image observation, temperature measurements, exhaust gas analyses and spectral measurements. We report the experimental results of developed several geometric types of the small scale catalytic combustors in order to investigate the catalytic combustion phenomenon in the combustor and to obtain the knowledge of the effective operation conditions for the TPV generation system.

\section{Experimental Apparatus and Method}

\subsection{Catalytic Combustor}

Schematic drawings of the catalytic combustors fabricated in the present experiment are shown in Figs.1. The combustor was composed of rectangular stainless steel (thickness is $10 \mathrm{~mm}$ ) hollowed a rectangular shape, two stainless steel side plates and a borosilicate glass for the observation window. There were three geometric types of the combustors (combustors A, B and C). The chamber volume of the combustor A was $5000 \mathrm{~mm}^{3}(10 \mathrm{~mm}$ $\times 50 \mathrm{~mm} \times 10 \mathrm{~mm})$, that of combustor $B$ was $2500 \mathrm{~mm}^{3}(10 \mathrm{~mm} \times 25 \mathrm{~mm} \times 10 \mathrm{~mm})$ and that of combustor $C$ was about $5000 \mathrm{~mm}^{3}(6 \mathrm{~mm} \times 84 \mathrm{~mm} \times 10 \mathrm{~mm})$. The hollowed rectangular space was used as a combustion chamber and the sphere-shaped Pt / Alumina pellet catalysts (Nikki Universal Inc., NS catalyst, diameter $=2.5-3.3 \mathrm{~mm}$, catalyst amount $=0.45 \mathrm{wt} \%$ ) were packed in that space. The advantages of using the pellet catalysts are that we can make a catalytic combustor easily by charging pellet catalysts in the combustion chamber and that we can change a characteristic of the catalytic combustor easily by replacing with other pellet catalysts.

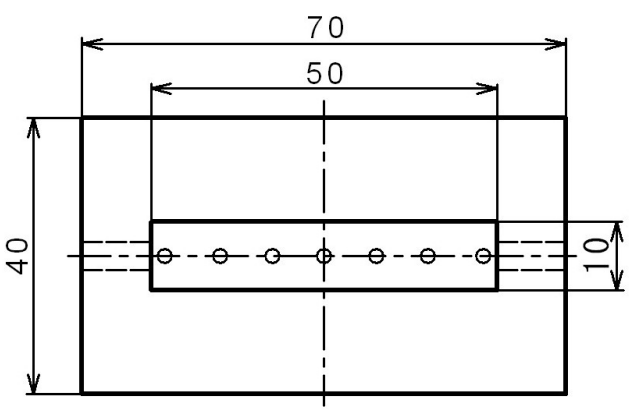

(1)Combustor A

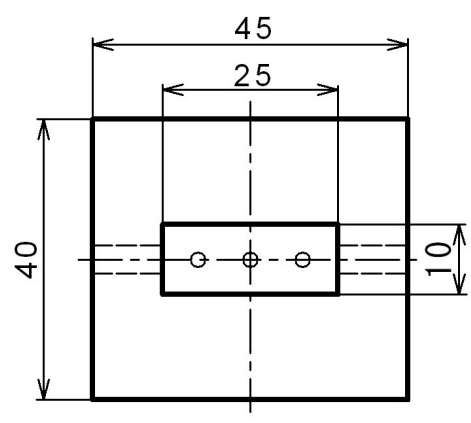

(2)Combustor B

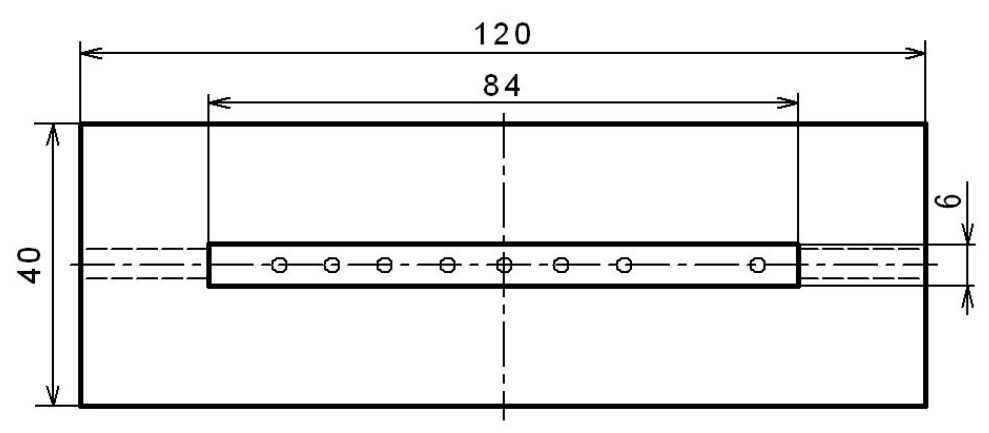

(3)Combustor C

Figs.1 Schematic drawings of the catalytic combustors.

\subsection{Experimental Apparatus}

The experimental apparatus employed in the present study is shown in Fig.2. $\mathrm{CH}_{4}$ was 
used for fuel and air $\left(\mathrm{O}_{2} ; 21 \%, \mathrm{~N}_{2} ; 79 \%\right)$ was used for oxidant. The flow rate of $\mathrm{CH}_{4}$ and air was controlled by mass flowmeters, and $\mathrm{CH}_{4}$ and air were premixed in the mixing section. The premixed gas of $\mathrm{CH}_{4}$ and air was supplied from the inlet port to the combustion chamber and the burned exhaust gas was exhausted from the outlet port to the vent. Air flow rate $\left(\mathrm{Q}_{\text {Air }}\right)$ was changed from 1.0 to $5.0 \mathrm{~L} / \mathrm{min}$. Equivalence ratio $(\phi)$ was changed from 0.67 to 1.00 . The ignition of the catalytic combustion was conducted by supplying heat to the combustion chamber through the irradiated ray of a halogen heater.

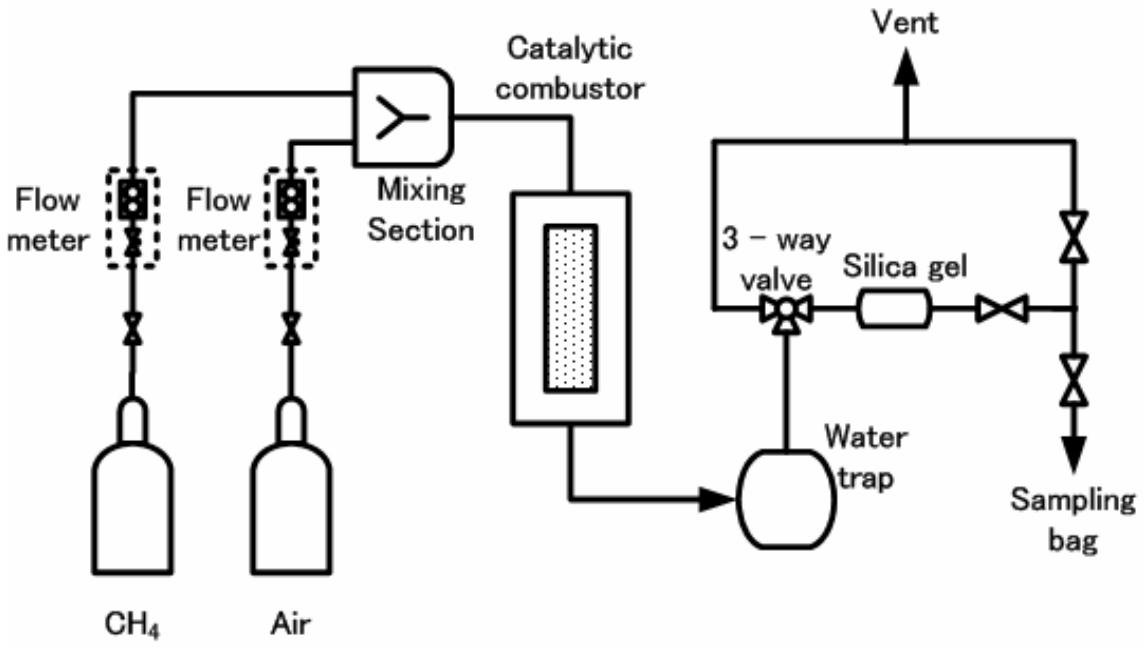

Fig.2 Schematic drawing of the experimental apparatus.

\subsection{Direct Image Observation, Temperature and Exhaust Gas Measurements}

The direct image observation of the combustor, the temperature measurements in the combustion chamber and the exhaust gas measurements were conducted to evaluate the performance of the developed catalytic combustors. The direct images of the catalytic combustion from the observation window were taken by a digital camera. Temperature in the combustion chamber and that in the exhaust gas were measured by $\mathrm{K}$ type contact thermocouples (diameter: $1 \mathrm{~mm}$ ) at the several positions of the combustion chamber. The exhaust gas was collected in a sampling bag from the outlet port of the combustor after removing water vapor through the water trap and silica gel. The collected gas was measured by the gas chromatograph (Varian Inc., Micro GC CP4900) in order to investigate the composition of the exhaust gas, especially the concentration of unburned $\mathrm{CH}_{4}$ and $\mathrm{CO}$. In the present study, $\mathrm{H}_{2}, \mathrm{O}_{2}, \mathrm{~N}_{2}, \mathrm{CH}_{4}, \mathrm{CO}, \mathrm{CO}_{2}$ and $\mathrm{C}_{2} \mathrm{H}_{6}$ are detected by the gas chromatography. Column CP740114/M5A (Varian Inc., length: $10 \mathrm{~m}$ ) and Ar carrier gas were used for detecting $\mathrm{H}_{2}, \mathrm{O}_{2}, \mathrm{~N}_{2}, \mathrm{CH}_{4}$ and CO. Column CP740124/PPQ (Varian Inc., length: $10 \mathrm{~m}$ ) and $\mathrm{He}$ carrier gas were used for detecting $\mathrm{CO}_{2}$ and $\mathrm{C}_{2} \mathrm{H}_{6}$. The combustion efficiency of the combustor can be calculated by the residual concentrations of $\mathrm{CH}_{4}, \mathrm{CO}$, $\mathrm{C}_{2} \mathrm{H}_{6}$ and $\mathrm{H}_{2}$ in the flue gas measured by the gas chromatography.

\subsection{Ultraviolet and Visible Spectral Measurements}

Ultraviolet and visible spectral measurements were conducted in order to investigate whether the gas phase reaction occurred or not in the catalytic combustor. The conditions of catalytic combustion can be classified as surface chemical reaction or parallel reaction of surface chemical reaction and gas phase reaction. The gas phase reaction is important to improve the combustion efficiency in the catalytic combustor because of its high reaction rate. However, it is uncertain that gas phase reaction occurred or not in the developed pellet catalytic combustors. In general, although it is difficult to discriminate the surface chemical reaction from the gas phase reaction, we observed the local chemiluminescence of $\mathrm{OH}^{*}$, $\mathrm{CH}^{*}$ and $\mathrm{C}_{2}{ }^{*}$ and tried to confirm the gas phase reaction in the combustor. The emitted light 
from the combustor was collected by the Cassegrain optical system [6]. The collected light was transferred to the ultraviolet and visible spectrometer (Oriel Inc., MS257) through the optical fiber and detected by the CCD camera with the image intensifier (ANDOR Inc., DH520-18F-04). The focus of the Cassegrain optical system was set on the surface point of the pellet catalyst that was moved in the horizontal plane by the 3D traverser. Measurement positions were selected at three points as follows; the catalyst surface where the strong emission occurred, the catalyst surface where the weak emission occurred and the gas phase region where there were not the pellet catalysts. The gas phase region where there were no pellet catalysts was specially prepared for the spectral measurements at the center of the combustor.

\subsection{Infrared Spectral Measurements}

Infrared spectral measurements were conducted in order to evaluate the infrared ray emitted from the combustor. The emitted ray from the combustor was collected by the Cassegrain optical system and it was focused onto a pinhole in front of the optical fiber. The collected light was transferred to the Fourier transfer infrared spectrometer (ARCoptix Inc., ARCspectro-NIR) through the optical fiber. The focus of the Cassegrain optical system was set at the surface point of the pellet catalyst that was moved in the horizontal plane by the $3 \mathrm{D}$ traverser.

\section{Experimental Results and Discussions}

\subsection{Temperature and Exhaust Gas Measurements}

Figures 3 show the temperature distributions in the combustion chamber. The flow rate, the fuel concentration and the structure of the combustor affected the emitting states of the combustor. With the decrease of equivalence ratio, the strong emission area in the combustion chamber decreased in all cases of combustors A, B and C. Moreover, the temperature in the upper region (from $\mathrm{TC} 1$ to $\mathrm{TC} 4$ ) decreased and the temperature gradient in the upper region decreased in all cases of combustors $\mathrm{A}, \mathrm{B}$ and $\mathrm{C}$. These results indicate that the intensity of the catalytic combustion reaction decreased with the decrease of the equivalence ratio because of the heat generation reduction. The strong emission area corresponds to the region where the catalytic combustion reaction is active and the temperature is relatively high. Interestingly, the strong emission area, in other words, the high temperature region, moved to the downstream region in the cases of combustor $\mathrm{A}$ and $\mathrm{C}$ at the equivalence ratio of 0.67 although the ignition didn't occur in the case of combustor B. The highest temperature in the combustion chamber of combustor $\mathrm{C}$ decreased in comparison with that of combustor A because of the heat loss increase caused by the narrow width.

Figures 4 show the residual concentrations of $\mathrm{H}_{2}, \mathrm{CO}$ and $\mathrm{CH}_{4}$ in the exhaust gas. Figure 5 shows the combustion efficiency of the three combustors that was calculated by the residual concentrations of $\mathrm{CH}_{4}, \mathrm{CO}, \mathrm{C}_{2} \mathrm{H}_{6}$ and $\mathrm{H}_{2}$ in the flue gas. With decrease of the equivalence ratio, the combustion efficiency of the combustor decreased and the decrease of $\mathrm{CO}$ concentration as well as the increase of $\mathrm{CH}_{4}$ concentration were observed. These results show that the equivalence ration relates to the oxidation rate of $\mathrm{CH}_{4}$. According to the theory of catalytic reaction rate, it is known that the oxidation reaction rate of hydrocarbons on $\mathrm{Pt}$ catalyst is the first order relation to the fuel concentration [7]. The deposition of intermediate products, such as $\mathrm{CO}$, likely occurred under near stoichiometric condition. Therefore, the oxidation rate of $\mathrm{CH}_{4}$ decreased and the concentration of $\mathrm{CO}$ was also decreased simultaneously with the decrease of the equivalence ratio. On the other hand, we can consider that the combustor length is related to the reduction of $\mathrm{CO}$ by comparing the residual $\mathrm{CO}$ concentration in the cases of combustors $\mathrm{A}$ and $\mathrm{B}$ at $\phi=0.86$ and 0.95 in 


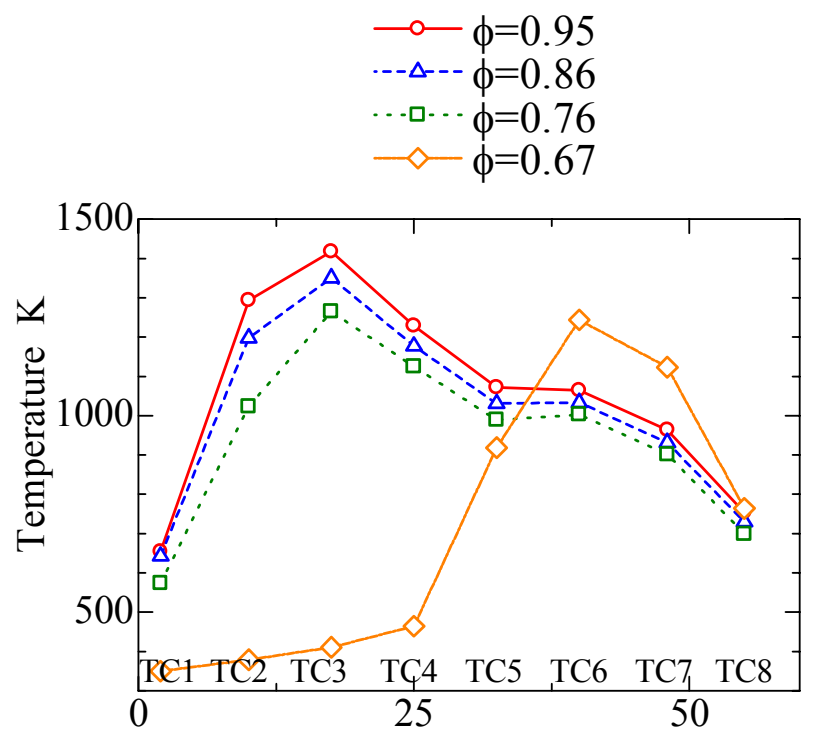

Distance from the inlet $\mathrm{mm}$

(1) Combustor A

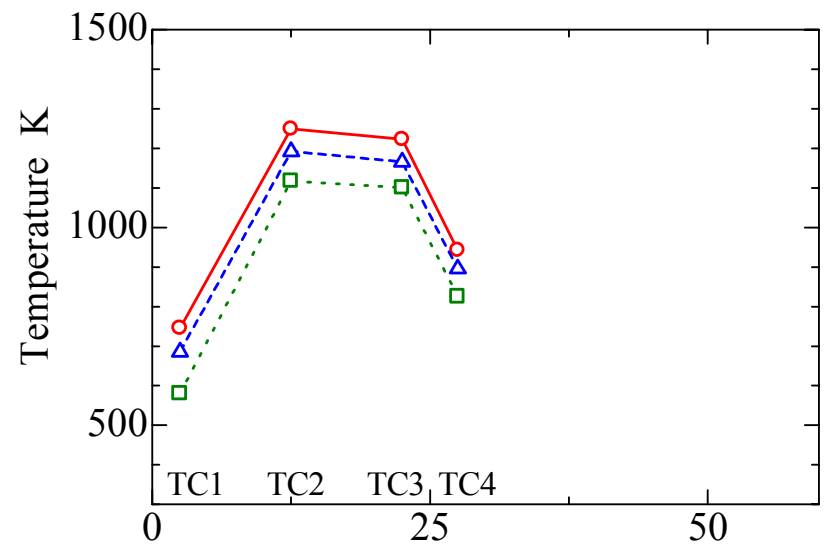

Distance from the inlet $\mathrm{mm}$

(2) Combustor B

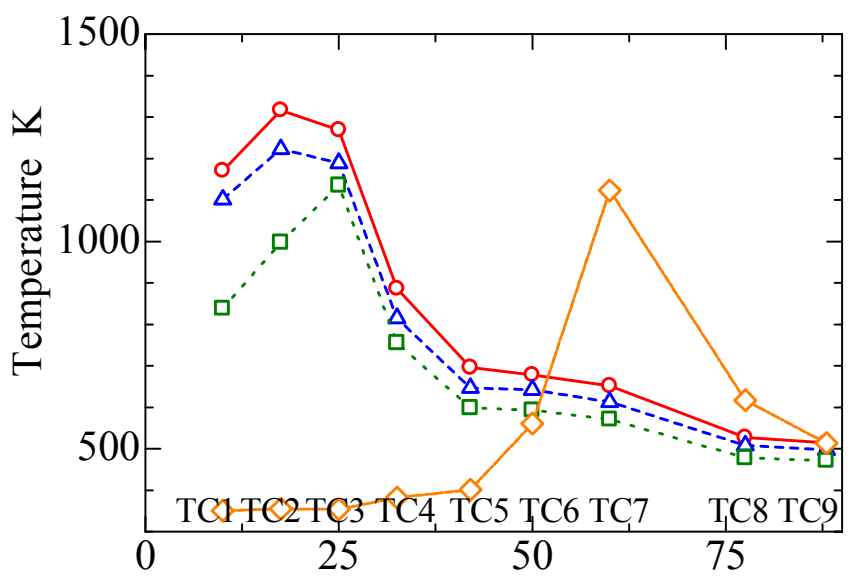

Distance from the inlet $\mathrm{mm}$

(3) Combustor $\mathrm{C}$

Figs.3 Temperature distributions depending on the combustor types and the equivalence ratio: $\mathrm{Q}_{\text {Air }}=5 \mathrm{~L} / \mathrm{min}$. 


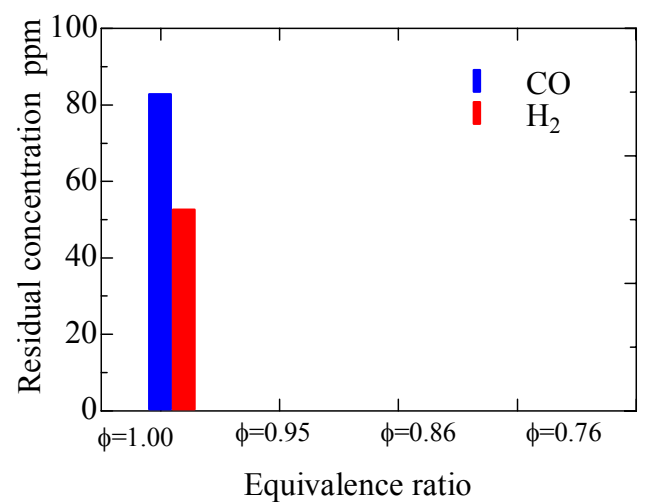

(1) Combustor A

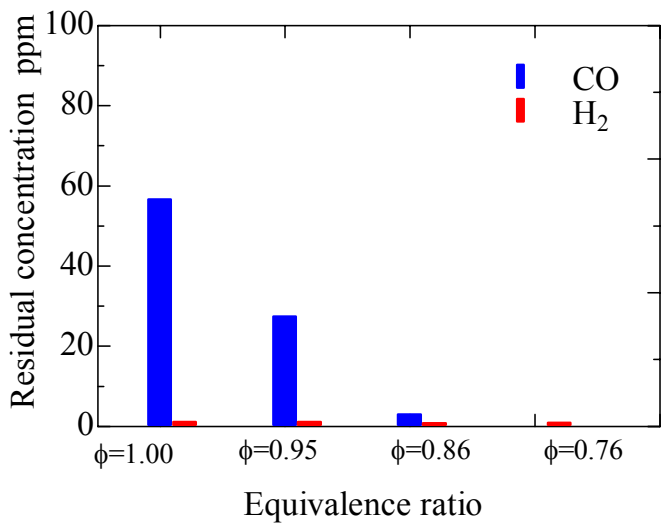

(2) Combustor B

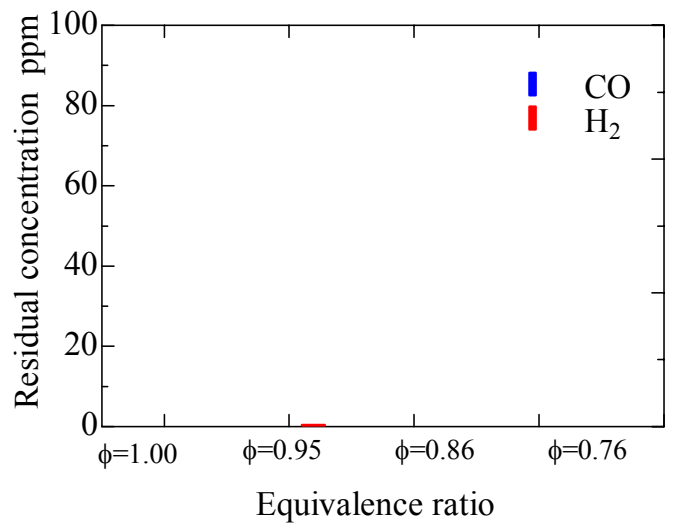

(3) Combustor $\mathrm{C}$

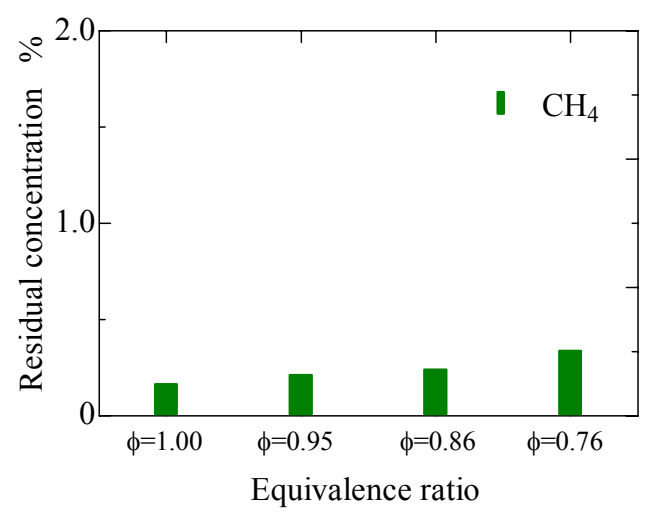

(1) Combustor A

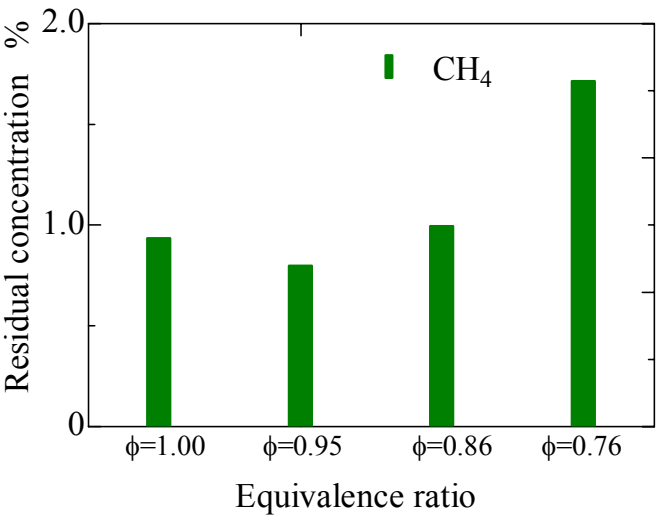

(2) Combustor B

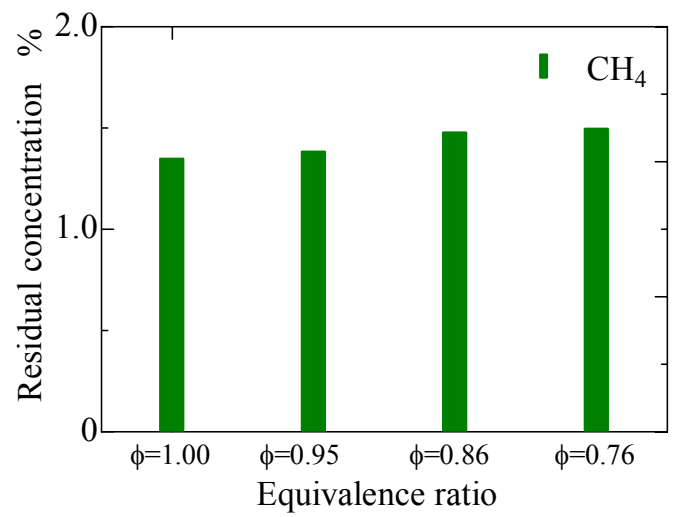

(3) Combustor $\mathrm{C}$

Figs.4 Residual concentrations of $\mathrm{CO}, \mathrm{H}_{2}$ and $\mathrm{CH}_{4}$ in the exhaust gas at $\mathrm{Q}_{\text {Air }}=5 \mathrm{~L} / \mathrm{min}$ depending on the combustor types and the equivalence ratio. 


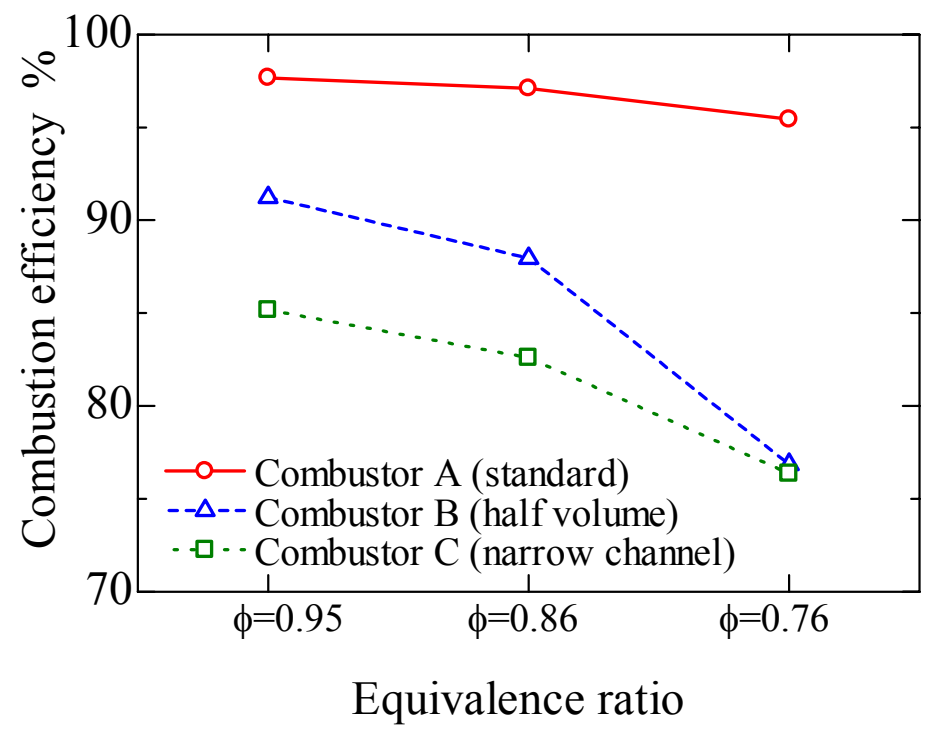

Fig.5 Combustion efficiency of the combustors at $\mathrm{Q}_{\text {Air }}=5 \mathrm{~L} / \mathrm{min}$.

Figures 4. In the case of combustor $\mathrm{C}$ the residual $\mathrm{CO}$ concentration is smaller in comparison with those in the cases of combustors $\mathrm{A}$ and $\mathrm{B}$. However, the combustion efficiency of combustor $\mathrm{C}$ is much smaller than those of combustors $\mathrm{A}$ and $\mathrm{B}$. The experimental results concerning with the temperature distributions and the exhaust gas measurements are summarized as follows; the high temperature region in the combustor corresponds to the strong emission area and the catalytic reaction has activated in the high temperature region. The temperature distributions are very much dependent on the geometry of the combustor as well as the equivalence ratio. The residence time after the high temperature region was related to the residual CO concentration. Hence, there are the optimal width and length of the combustor geometry for high combustion efficiency and the reduction of the residual $\mathrm{CO}$ concentration.

\subsection{Ultraviolet and Visible Spectral Measurements}

Figure 6 shows the experimental results of ultraviolet and visible spectral measurements employing the combustor A. The measurement results at the catalyst surface show that the continuous light emission was observed in the visible spectral range and the difference between the strong emission and the weak emission corresponds to the difference of the intensity of the continuous emission. The measurement results at the gas phase region in the combustor show that there was weak continuous emission and little chemical reaction was observed involving local spectral chemiluminescence in comparison with the premixed flame of air and methane. The gas phase region where there were no pellet catalysts was specially prepared for the spectral measurements at the center of the combustor. These experimental results show that the gas phase reaction involving local spectral chemiluminescence scarcely occurred at both the surface of the pellet catalysts and the gas phase region in comparison with the premixed flame of air and methane. Hence, the surface chemical reaction was predominant in the developed catalytic combustor and the emission from the combustor was originated from the thermal radiation of the pellet catalysts heated at the high temperature. 


\subsection{Infrared Spectral Measurements}

Figure 7 shows the experimental results of the infrared spectral measurements employing the combustor A. These results show that the near infrared ray (NIR) was generated from the developed catalytic combustor and the emissive intensity was enhanced with the increase of the amount of the fuel supply. The experimental results show that our proposed concept for generating NIR by the pellet catalyst combustor was applicable to the TPV system and the pellet catalysts in the combustor could play a role of the emitter for the TPV generation system.

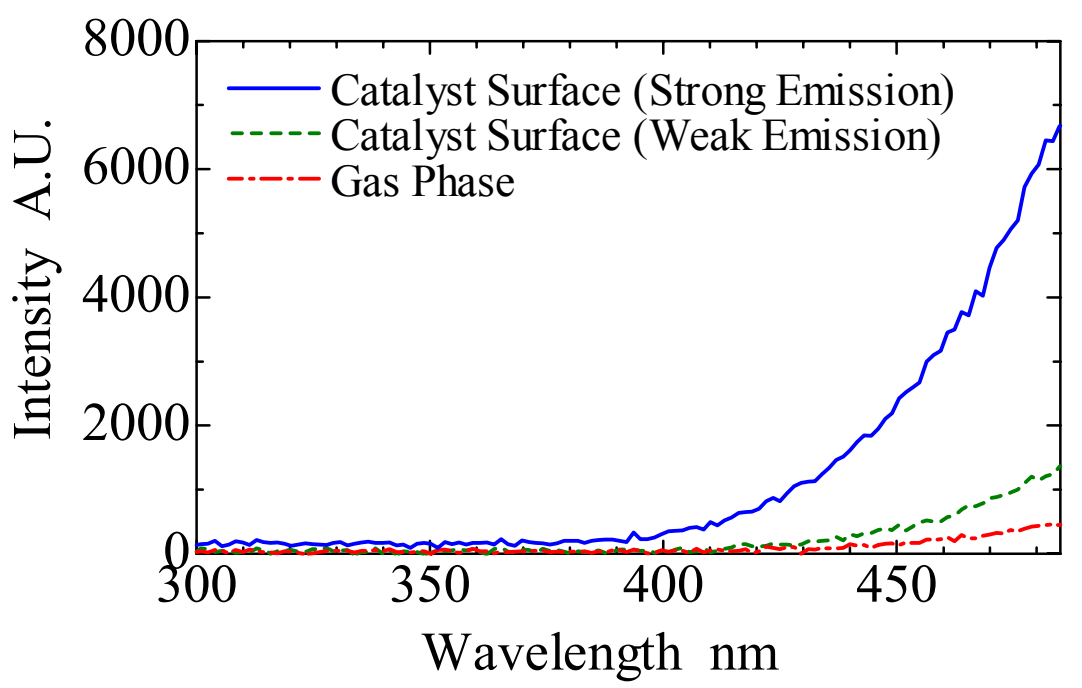

Fig.6 Ultraviolet and visible spectral ray emitted from the catalytic combustor $: \mathrm{Q}_{\text {Air }}=5 \mathrm{~L} / \mathrm{min}, \phi=0.95$.

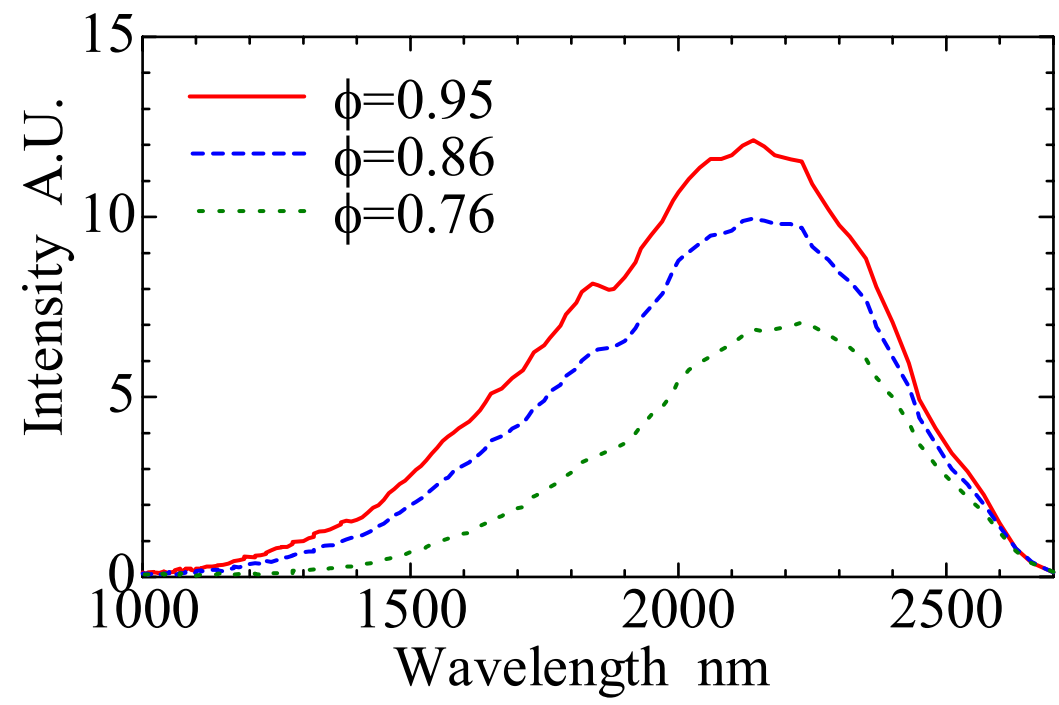

Fig.7 Infrared spectral ray emitted from the catalytic combustor.

\section{Conclusions}

Small scale catalytic combustors applying the pellet catalysts were developed in order to investigate the catalytic combustion phenomenon and the effective operation conditions 
for the TPV generation system. In the present paper, we investigated the performance of several geometric types of the combustors as well as the mechanism of catalytic combustion and evaluated them as the heat source/emitter for the TPV system. The main findings were as follows:

(1) There were the optimal width and length of the combustor geometry for the high combustion efficiency and the reduction of the residual CO concentration. The combustor A is the best for the combustion efficiency and the reduction of the residual $\mathrm{CO}$ concentration among the three combustors employed in the present study.

(2) The surface chemical reaction was predominant and the gas phase reaction involving local chemiluminescence scarcely occurred in the developed catalytic combustor.

(3) The emitted thermal radiation from the pellet catalysts was enhanced with the increase of the fuel supply under the present experimental conditions.

(4) The pellet catalysts in the developed combustor could play a role of an emitter for near infrared ray generation.

\section{References}

(1) Chia, L. C. and Feng, B., The Development of a Micropower (Micro-Thermophotovoltaic) Device, Journal of Power Sources, Vol. 165, No. 1, 2007, pp. 455-480.

(2) Okamasa, T., Lee, G. -G., Suzuki, Y., Kasagi, N. and Matsuda, S., Micro Catalytic Combustor Using High-Precision Ceramic Tape Casting, Journal of Micromechanics and Microengineering, Vol. 16, No. 9, 2006, pp. 198-205.

(3) Kumano, T. and Hanamura, K., Energy Conversion from Fossil Fuel into Spectral-Controlled Radiation for TPV by Super-Adiabatic Combustion in Porous Quartz Glass, Proceedings of 2007 ASME-JSME Thermal Engineering Summer Heat Transfer Conference, HT2007-32509, ASME, 2007.

(4) Nakagawa, N., Ohtsubo, H., Waku, Y. and Yugami., H., Thermal Emission Properties of $\mathrm{Al}_{2} \mathrm{O}_{3} / \mathrm{Er}_{3} \mathrm{Al}_{5} \mathrm{O}_{12}$ Eutectic Ceramics, Journal of European Ceramic Society, Vol. 25, No. 8, 2005, pp. 1285-1291.

(5) Pan, J. F., Huang, J., Li, D.T., Yang, W.M., Tang, W. X. and Xue, H., Effects of Major Parameters on Micro-combustion for Thermophotovoltaic Energy Conversion, Applied Thermal Engineering, Vol. 27, No. 5-6, 2007, pp. 1089-1095.

(6) Akamatsu, F., Wakabayashi, T., Tsushima, S., Katsuki, M., Mizutani, Y., Ikeda, Y., Kawahara, N. and Nakajima, T., The Development of a Light-collecting Probe with High Spatial Resolution Applicable to Randomly Fluctuating Combustion Fields, Measurement Science and Technology, Vol. 10, No. 12, 1999, pp. 1240-1246.

(7) Prasad, R., Kennedy, L.A. and Ruckenstein, E., Catalytic Combustion, Catalysis Reviews: Science and Engineering, Vol. 26, No. 1, 1984, pp. 1-58. 